\title{
The Phantom Leaf Effect and Its Implications for Near-Death and Out- of-Body Experiences
}

\author{
James C. Pace, R.N., D.S.N., M.Div. \\ Deborah L. Drumm, R.N. \\ Vanderbilt University
}

\begin{abstract}
The phantom leaf effect seen in Kirlian photography may help researchers better understand near-death and out-of-body experiences. While the process responsible for the phantom leaf effect is unknown, variations of Rupert Sheldrake's morphogenetic field theory offer three explanations for this phenomenon. Each of these variations has different implications for neardeath and out-of-body experiences.
\end{abstract}

The phantom leaf phenomenon of electrical or Kirlian photography may provide an important clue to the interpretation of both near-death and out-of-body experiences (NDEs and OBEs). In this paper, we explain the phantom leaf effect, explore a possible relationship to humans, and examine this effect's importance for NDEs and OBEs. We describe three processes potentially responsible for the phantom leaf effect and the implications for NDEs and OBEs of each process.

James C. Pace, R.N., D.S.N., M.Div., is a hospice chaplain at Alive Hospice and Associate Professor of Nursing at Vanderbilt University. Deborah L. Drumm, R.N., is a graduate student in Psychiatric Nursing at Vanderbilt University. Reprint requests should be addressed to Ms. Drumm at 713 Richfield Drive, Nashville, TN 37205. 


\section{Phantom Effects and Implications}

The phantom leaf effect is obtained by cutting away up to 50 percent of a leaf and then photographing it in the dark using electricity instead of light: the entire leaf, including the absent portion, appears in the photograph, sometimes in such exquisite detail that the spine, veins, and skin of the absent portion are readily apparent. Kirlian photographs of leaf phantoms have been obtained using many different kinds of leaves, including maples (Krippner and Rubin, 1974), creeping Charlies (Johnson, 1975), potato plants ( $\mathrm{H}$. Oldfield, personal communication, autumn, 1990), and sultanas (G. Gay, personal communication, autumn, 1990). Those who have photographed the phenomenon have reported frequencies of from one appearance in several hundred photographs (Johnson, 1975) to 85 appearances in 120 photographs (J. Hubacher, personal communication, autumn, 1989).

The phantom leaf effect is neither a double exposure nor a photographic trick; precautions are routinely taken to ensure that an image cannot occur by mistake. For example, it is standard procedure to cut the leaf immediately before it is smoothed onto the emulsion (photographs are by direct contact) to eliminate the possibility that images could result from moisture prints. In the early 1970 s, the credibility of the first phantom photographs was jeopardized by the practice of cutting the leaf while on the emulsion, but that practice was abandoned in the mid-1970s.

Is there a related phenomenon in humans? For many years, healthcare professionals have noted the occurrence of phantom limb sensations in amputees, in which amputees complain of itching or pain in their absent extremities. Current theories cite exposed and stimulated nerve endings as the cause of these sensations. But some amputees have complained of these sensations for more than 20 years, long after the nerve endings have healed and stimulations have ceased.

There are at least two reports of the photographing of phantom limbs in humans. Jack Worsley (1973) and Joanne Cusack (1989), working in different countries and without prior knowledge of each other, both concluded that phantom limbs can only be photographed during phantom limb pain. While those investigators did not conduct formal studies, and did not monitor or report critical factors such as applied voltage and frequency of appearance, this information implies that, under certain conditions, a photographable phantom effect may exist in humans as well as in plants.

In NDEs and OBEs, individuals often claim that they experience existence separated from the physical body. Since objective evidence of 
this out-of-body state is particularly difficult to find, the credibility of the separation hypothesis remains in question. Despite obvious differences in genetic makeup and consciousness between plants and humans, the recorded image of a leaf section where no physical counterpart exists may be visual evidence of a nonphysical dimension, a state of at least partial independence from the body.

Since the phantom half of the leaf may be projected from the remaining physical portion, the assumption cannot necessarily be made that the phantom is distinct and separate from its physical counterpart. Nevertheless, the fact that the photographed image extends beyond the leaf's cut borders indicates existence of something at least partially independent from the physical body. If such projections exist in human beings, they could account for both the realness and the veridicality of NDEs and OBEs.

\section{Three Explanations for the Phantom Leaf Effect}

Three hypotheses offer explanations for the phantom leaf effect. Each hypothesis has different ramifications for NDEs and OBEs.

\section{Harmonic Projection from a Cellular Blueprint}

One hypothesis postulates that the phantom section may be projected from a blueprint in the cellular components of the material half of the leaf, perhaps in the deoxyribonucleic acid (DNA). This is the contention of researchers Harry Oldfield and Roger Coghill (1988):

We think that cells broadcast via the resonance of their DNA paired bases' hydrogen bonds and transmit morphogenetic information to adjacent cells. The Kirlian phantom leaf effect is, therefore, produced by intensifying, rather than destroying, the relevant specific frequencies, and these are reproduced by downward harmonics in the visible light frequency range, and captured on photographic film. If the correct frequency is broadcast by a large number of cells it becomes a strong signal, which bonds the whole cellular structure of an organism together. It embodies a morphogenetic field. (p. 116)

Elsewhere, they state: "Radiation of waves at cellular frequencies, we believe, is how the Kirlian photography can produce the phantom leaf effect" (1988, p. 122) 
This interpretation does not permit extrapolation beyond the hydrogen bonds of DNA paired bases. It neither supports nor contradicts the separation hypothesis of NDEs and OBEs. All that can be assumed is that leaves either radiate encoded oscillations, perhaps similar to the amphibian stump currents described by Joseph Vanable, Lester Hearson, and M.E. McGinnis (1983), or that the material blueprint in the cellular matter of the leaf is somehow able to direct applied electrical current into a leaf pattern. We might also speculate that the same process might exist actually or potentially in other living things, including humans.

This theory by itself does not adequately explain NDEs and OBEs. In electrical photography, the additional energy needed to intensify the broadcast of waves at the cellular level, and thus to project the phantom, is generated by currents from the photographic machine. It is possible that changes in the brainwave patterns or surges in the brain's electrical activity could provide the energy to intensify cell broadcasts and induce out-of-body projections in living persons, but what could produce strong electrical emissions in the clinically dead?

Janusz Slawinski (1987) described a "death flash" of electromagnetic radiation as the moment of passing, but it is questionable whether broadcast waves from cells deteriorating and suffering from anoxia or hypercapnea could be strong enough to produce a human phantom, even if reinforced by some type of radiation from cellular breakdown. And if they could do so, it is questionable whether the emission of such radiation could last more than a matter of seconds. What then should be made of vivid NDE accounts such as that of Ricky Bradshaw, who was reported clinically dead for over an hour, during which time he apparently experienced an NDE (Atwater, 1988)?

\section{Projection from a Morphogenetic Field}

A second possible explanation of phantom phenomena assumes that there is something that acts as a blueprint for the DNA itself, channeling the molecules of genetic material into their proper positions and directing the growth of the plant from beginning to end. Rupert Sheldrake (1981) posited the existence of such an underlying multidimensional form in his theory of morphogenetic fields:

The morphogenetic germ is part of the system-to-be. ... The field is not yet "occupied" or "filled out"; it contains the virtual form of the 
final system, which is actualized only when all its material parts have taken up their appropriate places. (p. 76)

By morphic resonance the form of a system, including its characteristic internal structure and vibrational frequencies, becomes present to a subsequent system ... superimposes itself on the latter. (p. 96)

In the case of phantom leaves, such a morphogenetic form may underlie the entire plant, or it could be a "germ" of spatial coordinates and polarities that allows the projection of the developing plant, much the way the indistinct pattern in the film beyond a hologram creates the unfolded three-dimensional image. The interpretation of the field as a germ of spatial coordinates may be closer to Sheldrake's intention: "Morphogenetic fields in general are not precisely defined, but are given by probability distributions. ... The structures of morphogenetic fields will henceforth be referred to as probability structures" (1981, p. 83).

Physicist David Bohm (1980) hinted at the existence of morphogenetic germs in his explanation of the quantum properties of the electron:

The form of the quantum potential can dominate... Information contained within quantum potential will determine the outcome of the quantum process. Indeed, it is useful to extend this idea to what is called active information. The basic idea of active information is that a form, having very little energy, enters into and directs a much greater energy. (p. 93)

If Sheldrake's theory is correct, the phantom leaf effect may be produced by a spatial blueprint or seed form that momentarily translates applied electrical currents into a partial projection of a leaf, as under different circumstances it might channel atoms and molecules into the developing plant. This preexisting morphogenetic form would be capable of sustained independence from its material product. Applied to NDEs and OBEs, this hypothesis suggests separability of a formative structure, with all the potential capabilities of the manifested entity.

\section{Combined Morphogenetic Field-Harmonics Hypothesis}

A third possible explanation of the phantom leaf effect combines Sheldrake's idea of a preexisting form with the harmonics theory of Oldfield and Coghill. In this interpretation, the preexisting mor- 
phogenetic field incites the resonance of those DNA paired bases' hydrogen bonds by which cells broadcast their specific frequencies. The paired bases' hydrogen bonds serve as organic transistors that tune in to and project the morphogenetic frequency. If cell resonances are programmed to span out inches or feet away from the plant, they could create an electromagnetic field or other wave pattern that releases encoded information as electrical currents are applied, analogous to the illumination of an interference pattern by a laser beam. This hypothesis incorporates the multidimensionality of Sheldrake's theory with Oldfield's mechanisms by which field forms could incite matter. As in Sheldrake's theory by itself, in this combined hypothesis the preexisting structure would be capable of independence from the physical body.

The morphogenetic theory, then, that offers the most comprehensive interpretation of phantom leaf phenomena also accounts for separability, and therefore of veridicality, in NDEs and OBEs. Furthermore, the curving epigenetic landscape that Sheldrake indicated may be associated with the underlying morphogenetic form could allow for a multidimensionality as described by Gordon Greene (1983), Hornell Hart (1953), and Austin Farrer (1965). Many of the strange perceptual effects associated with OBEs and NDEs make sense in that context; the dynamic curvature of four-dimensional space could explain, for example, the 360-degree vision, ability to see around corners, and sensation of having a spherical or balloon-like body sometimes reported in OBEs and NDEs.

Kenneth Ring (1980) wrote that the NDE could be explained by an assimilation of the holographic paradigm of Bohm and Karl Pribram into a parapsychological framework: "In my view, these two components together can furnish an account of virtually every aspect of the core experience" (p. 220). Ring's parapsychological-holographic model is compatible with the morphogenetic interpretation of phantom phenomena.

The holographic paradigm is based on interconnected dimensional layers that Bohm called the universal matrix, out of which frequencies are projected into three-dimensional entities. The morphogenetic theory also presents a multidimensionality that projects a threedimensional form through the effects of morphic resonance. Indeed, Bohm described reality as an enfoldment, or series of everpresent existences or enfolded similar ensembles: "There's a natural sequence like this series of Chinese boxes within boxes. And the present moment might be called the box which contains all these previous moments as its content" (Bohm and Weber, 1978, p. 45). 
This enfoldment concept is reminiscent of the leaf form under the leaf, the phantom beyond the material counterpart. In fact, Sheldrake (1988) quoted Bohm as making that precise point about the enfoldment process: "Past forms would tend to be repeated or replicated in the present, and that is very similar to what Sheldrake calls a morphogenetic field and morphic resonance" (p. 305). Thus both Bohm and Sheldrake have noted the connection between their respective theories; if the morphogenetic theory helps explain phantom phenomena, then so may the holographic theory as well.

\section{Conclusion}

The phantom leaf effect may be the closest thing we have to recorded visual evidence of nonphysical existence. By studying phantom phenomena, we may come closer to understanding human out-of-body and near-death experiences, and closer to understanding what happens at the moment of death. Whatever causes phantom leaf effects may also be involved in out-of-body projections of the NDE. We argue for considering morphogenetic fields as a factor in both phenomena.

Of course, the concept of morphogenetic fields is still speculative, and we have no device for detecting them. However, despite their unproven existence, many natural occurrences suggest their presence. The very fact of phantom leaves implies some kind of nonmaterial field-like structure reproducible in plants. As for the phantom leaves, there have been few attempts to define their essence, and the phantom effect is reproducible only after many trials and not always on the same coordinates. While both the phantom leaf effect and the morphogenetic field hypothesis remain open to question, they seem mutually supportive. We suggest that further research into phantom phenomena, in the light of morphogenetic field theory, may yield observations relevant to our understanding of life and the moment of death.

\section{References}

Atwater, P.M.H. (1988). Coming back to life: The after-effects of the near-death experience. New York, NY: Dodd, Mead.

Bohm, D. (1980). Wholeness and the implicate order. London, England: Routledge and Kegan Paul.

Bohm, D., and Weber, R. (1978). The enfolding-unfolding universe: A conversation with David Bohm conducted by Renee Weber. ReVision, 1, 24-51. 
Cusack, J. (1989, April). Kirlian photography: The mind energy connection. Tap the Source, p. 8.

Farrer, A. (1965). Saving belief. New York, NY: Morehouse-Barlow.

Greene, F.G. (1983). Multiple mind/body perspectives and the out-of-body experience. Anabiosis: The Journal of Near-Death Studies, 3, 39-62.

Hart, H. (1953). The psychic fifth dimension. Journal of the American Society for Psychical Research, 47, 3-32.

Johnson, K. (1975). The living aura: Radiation field photography and the Kirlian effect. New York, NY: Hawthorne.

Krippner, S., and Rubin, D. (1974). Epilogue to the Anchor edition. In S. Krippner and D. Rubin (Eds.), The Kirlian aura: Photographing the galaxies of life (p. 194). New York, NY: Anchor/Doubleday.

Oldfield, H., and Coghill, R. (1988). The dark side of the brain: Major discoveries in the use of Kirlian photography and electrocrystal therapy. Longmead, England: Element Books.

Ring, K. (1980). Life at death: A scientific investigation of the near-death experience. New York, NY: Coward, McCann and Geoghegan.

Sheldrake, R. (1981). A new science of life: The hypothesis of formative causation. Los Angeles, CA: Tarcher.

Sheldrake, R. (1988). The presence of the past: Morphic resonance and the habits of nature. New York, NY: Times Books.

Slawinski, J. (1987). Electromagnetic radiation and the afterlife. Journal of Near-Death Studies, 6, 79-94.

Vanable, J.W., Hearson, L.L., and McGinnis, M.E. (1983). The role of endogenous electrical fields in limb regeneration. In J.F. Fallon and A.I. Caplan (Eds.), Limb development and regeneration. Part A: Proceedings of the third international conference on limb morphogenesis and regeneration (pp. 587-596). New York, NY: Alan R. Liss.

Worsley, J. (1973). Questions from the audience. In Krippner, S., and Rubin, D. (Eds.), Galaxies of life: The human aura in acupuncture and Kirlian photography. Proceedings of the first Western Hemisphere conference on Kirlian photography, acupuncture, and the human aura (pp. 145-154). New York, NY: Gordon and Breach/Interface. 